\title{
LES ASPECTS MÉTHODOLOGIQUES DES RAPPORTS ENTRE CONCEPTS DU SIGNE ET DU SYMBOLE DANS LA THÉORIE HISTORICO-CULTURELLE DE L.S. VYGOTSKI
}

\author{
Yury P. Zinchenko
}

L'Université d'État de Moscou Lomonossov

Moscou

Dans le texte, il s'agit de sources de la compréhension des concepts du signe et du symbole dans la théorie historico-culturelle ainsi que de leur rôle dans le développement de l'enfant et dans le processus de la médiation. On y parle aussi de limites de l'approche historico-culturelle en ce qui concerne la compréhension du signe et du symbole et d'autres concepts liés avec eux.

Mots clés: Vygotski, théorie historico-culturelle, signe, symbole, signification, instrument, médiation.

\section{Lev Vygotski (1896-1934),}

\section{fondateur de la théorie historico-culturelle}

Les concepts du signe et du symbole occupent une place importante dans la théorie historico-culturelle établie par Lev Vygotski, un psychologue russe très connu en Russie et ailleurs. La psychologie historicoculturelle est une conception du développement psychique de l'homme proposée dans les années 1920-30 par Vygotski et ses collègues Léontiev et Luria (Zinchenko, Tkhostov, Shilko, 2008). Cette école a été fondée au déclin de lâge d'argent ou de la Renaissance de la culture russe où il n'existait pas de frontières strictes entre la science et l'art, l'esthétique, la philosophie et même la théologie. Schpet, Lossev, Bakhtin, Florenski se spécialisaient en tous ces sphères au même temps. Vygotski lui-même nétait pas seulement psychologue, mais aussi critique de l'art, philosophe et méthodologue de la science. Un trait important de son approche était la tendance de l'intégration de différentes connaissances sur l'homme, des approches et méthodes diverses de l'étude du psychisme et du comportement de l'homme. 
Les idées de Vygotski ont donné naissance à bien des recherches, des expérimentations dans différents domaines de la psychologie et de sciences contigües. Les élèves et collègues de Vygotski (A.N. Léontiev, A.R. Louria, P.I. Zinchenko et d'autres) ont développé son approche et transmis ses idées à la génération contemporaine de psychologues en Russie. "Vygotski a introduit dans la recherche psychologique concrète l'idée de l'historicité de la nature du psychisme humain et celle du réaménagement des mécanismes naturels des processus psychiques au cours de lévolution socio-historique et ontogénétique. Vygotski interprétait ce réaménagement comme le résultat nécessaire de l’appropriation par l'homme des produits de la culture humaine au cours de ses contacts avec ses semblables", dit A.N. Léontiev de la contribution de son maître en sciences humaines (Léontiev, 1976).

Cette approche propose une compréhension de la représentation de la culture dans le psychisme. La thèse principal de la théorie est que la structure et le développement du psychisme sont engendrés par un environnement socioculturel qui pour Vygotski nest pas un facteur du développement mais une source du développement de la personnalité.

Il est à noter que la référence à la pensée de Vygotski n’a jamais été aussi importante dans les sciences humaines en Russie et surtout ailleurs qu'aujourd'hui. Les idées de Vygotski sur la médiation, la conscience, le développement du psychisme, le signe comme l'unité de la conscience trouvent sa deuxième naissance à un niveau différent de leur compréhension. Vygotski a développé de nombreux concepts sur lesquels l'école de la psychologie russe s'appuie presque entièrement depuis plusieurs dizaines d’années: fonctions psychiques supérieures, zone de développement le plus proche, intériorisation, signification, etc.

\section{L'art comme une source}

\section{de l'approche historico-culturelle}

Les idées de l'approche historico-culturelle en psychologie de Vygotski ont à sa base l'esthétique, sa monographie "Psychologie de l'art" (1925) et les idées de l'école française de psychologie (avant tout, la théorie de Piaget). En créant son approche, Vygotski a aussi réfléchi profondément sur la gestalt-théorie, la linguistique et la sémiologie (Saussure).

Il est important de tenir compte que Vygotski est venu à la psychologie de l'esthétique. C'est dans sa "Psychologie de l'art", qui représente un bilan de ses travaux de 1915-22, où il découvre que les affects de l'homme existent non pas seulement sous la forme des états subjectifs mais aussi sous la forme objective, externe. Dans ses premiers œuvres de critique de l'art, il a déjà cherché les esquisses de sa future psychologie historico-culturelle, de sa compréhension du rôle du signe dans lévolution de l'homme (Vygotski, 1925/2005).

La question principale à laquelle Vygotski cherche la réponse dans cet ouvrage est suivante: qu'est-ce qui transforme un œuvre en l'œuvre de l'art? La fonction principale de l'œuvre de l'art, ce n'est pas la transmission des connaissances ni l'expression des émotions. La fonction particulière de l'art est la catharsis qui Vygotski comprend autrement qu'Aristote. Pour lui la catharsis est une solution d'un certain problème personnel, la découverte d'une réalité la plus haute, la plus humaine. Comme le dit Vygotski, ce n'est que la catharsis qui a forcé un maitre ancien à mettre sur le Notre-Dame de Paris les images horribles et effrayantes des monstres, les chimères magnifiques sans lesquelles la cathédrale était inexistante.

Vygotski propose de différencier dans chaque œuvre d'art deux types des émotions: les émotions qui sont éveillées par le contenu et celles qui sont éveillées par la forme de cet œuvre. Ces deux types démotions se trouvent dans l'antagonisme éternel, et la loi de la réaction esthétique pour la fable ainsi que pour la tragédie est la même: elle contient en elle-même un affect qui se développe dans deux directions opposées et qui dans son point final trouve comme dans le court-circuit son anéantissement (Vygotski, 1925). Le nom de cette loi, selon Vygotski est la catharsis. Lartiste surmonte toujours le contenu par la forme, on peut l'observer comme dans la composition de la fable ainsi que de la tragédie.

\section{Signe dans le développement de l'enfant}

Vygotski a tenté de trouver un moyen empirique de la vérification de ses représentations sur le conditionnement culturel du psychisme. Pour cela, il a créé la méthode génétique de recherche: selon cette méthode, dans l'expérimentation on reconstitue le mécanisme hypothétique de la médiation culturelle d'une fonction psychique par un signe. Grâce à cela, il est devenu possible d'observer le processus de la naissance des fonctions psychiques chez l'homme dans "les conditions de laboratoire". 
Cette méthode a aidé à apporter la lumière sur le processus de la médiation chez l'homme et de la maîtrise des concepts artificiels.

Dans ses recherches expérimentales à la fin des années 1920, Vygotski mettait un enfant dans la situation où il avait comme la tâche de mémoriser quelque matière. Si cette tâche n’a pas dépassé ses capacités naturelles, il a crû que l'enfant la résolvait en utilisant des moyens naturels ou primitifs. Mais si la tâche a dépassé ses capacités naturelles, il a pensé qu'elle ne pouvait pas être résolue par un moyen primitif et naturel: "La tâche apparaît dans le processus de l'activité naturelle de l'enfant, mais sa solution demande de lui un moyen de détour ou l'usage d'un instrument. Si l'enfant crée la solution, il recourt à l'aide de signes, en faisant des nœuds, en comptant des dragées, en perçant ou en déchirant des papiers, etc. Nous concernons telle mémorisation qui est basée sur l'utilisation des signes comme un exemple typique de chaque technique culturelle du comportement. L'enfant résout la tâche intérieure à l'aide de moyens extérieurs; c'est dans ce fait que nous voyons le caractère le plus typique du comportement culturel", a écrit Vygotski dans le texte sur le développement culturel de l'enfant en 1928 (Vygotski, 1991, p. 9). Il en est venu à la conclusion que "les mémorisations s'appuyant sur des systèmes de différents signes seront aussi différentes en leurs structures. Le signe, ou le moyen auxiliaire d'une technique culturelle, forme de ce train-là un noyau de structure et de fonction qui détermine la composition et l'importance relative de chaque processus particulier. L'inclusion dans un processus du comportement d'un signe à l'aide duquel ce processus s'accomplit réorganise tout l'ordre des opérations psychologiques à la manière que l'inclusion d'un instrument réorganise tout l'ordre d'une opération de travail" (Vygotski, 1991, p. 10).

Sur un exemple de l'enfant qui mémorise à l'aide d'une carte géographique ou à l'aide d'un plan, d'un schéma, d'un abrégé Vygotski illustre le développement culturel de la mémoire. Il dit qu' 'on a de bonnes raisons pour croire que le développement culturel consiste à assimiler des techniques du comportement qui se basent sur l'usage et sur l'application des signes comme les moyens destinés à la réalisation de telle ou telle opération psychologique, que le développement culturel consiste en l'appropriation de tels moyens auxiliaires que les hommes ont crées dans le processus du développement historique et qui sont la langue, lécriture, le système numérique, etc. Nous en sommes persuadés non seulement grâce à des recherches psychologiques sur le développement de l'homme primitif, mais aussi grâce à des observations directes et immédiates auprès d'enfants" (Vygotski, 1991, p. 6).

\section{Médiation: signe et instrument}

Finalement, Vygotski a mis la médiation au centre de ses recherches et de son approche. Au début des années 1930, il s'est concentré sur létude de la structure de la conscience conditionnée par le signe. Il a montré que l'activité de médiation de l'homme réunissait deux processus: l'utilisation des instruments et celle des signes.

Le rôle de la médiation dans le développement psychique de l'homme a été étudié et est étudié à présent par ses élèves et adeptes dont les approches au problème de la médiation sont nombreuses et très différentes. Vygotski a stimulé les recherches sur le fonctionnement des instruments-moyens dans les processus de la pensée, de l'attention, de la mémorisation, etc.

De point de vue de Vygotski, le signe pour l'homme c'est avant tout un moyen social, en quelque sorte, un instrument psychologique. "Le signe qui se trouve à l'extérieur de l'organisme ainsi que l'instrument est séparé de la personnalité et, en fait, sert d'un organe ou d'un moyen social" (Vygotski, 1931/1984) Cependant, la différence la plus importante entre ces deux médiateurs consiste en leur orientation. Si l'instrument est orienté à l'extérieur parce qu'il sert à changer un objet, le signe ne change rien dans l'objet parce qu'il est un moyen de l'activité intérieure, de l'influence psychologique sur son comportement ou celui d'un autre. Donc, il est orienté à l'intérieur, à la maitrise de soi-même.

Au cours du développement de son approche Vygotski a changé son point de vue sur le rôle du signe dans le développement des fonctions psychiques. Si au début de ses recherches il croyait que les fonctions élémentaires de l'enfant avaient le caractère "naturel", génétique, c’est-à-dire elles nétaient pas encore conditionnées par les moyens-signes culturels, plus tard, en s'appuyant sur les recherches empiriques et les faits, il est venu à une autre conclusion importante: même les fonctions les plus élémentaires de l’enfant se caractérisent par la structure psychologique médiatisée, spécifiquement humaine.

Il précise sa compréhension du développement des fonctions psychiques de l'homme dans la loi suivante: "Chaque fonction psychique 
supérieure apparaît deux fois au cours du développement de l'enfant: d'abord comme activité collective, sociale et donc comme fonction interpsychique, puis la deuxième fois comme activité individuelle, comme propriété intérieure de la pensée de l'enfant, comme fonction intrapsychique" (Vygotski, 1933/1985, p. 111).

Le signe se présente le point d'intersection de la psychologie, de la philosophie et de la linguistique. Le signe pour Vygotski tout comme le mot est lélément principal dans le processus de la médiation. Cependant, il nanalyse pas de structure du signe. C’est la fonction du signe dans le développement des processus psychiques supérieurs qui lui est important. Comme dit Zavialoff, "Pour Vygotsky les signes ne sont pas seulement les représentations des objets-stimuli, ils sont aussi les moyens de médiation; mais ces formes symboliques du monde et les significations qui y sont associées sont externes, puis, dans l'échange, deviennent internes et c'est à ce niveau que se transforme la signification.. Le rapport entre les signes "internes" et les signes "externes" reflète non pas des structures linguistiques pré-données, mais la complexité des attitudes et actions cognitivo-émotionnelles des individus dans le cadre de la mémoire historique et aussi de ce que nous appelons la mémoire du vivant" (Zavialoff, 1998).

\section{Signification dans la vie de l'homme}

Selon Vygotski, la signification est un processus de la création et de l'utilisation des signes à l'aide desquels l'homme premièrement influence le comportement des autres, puis les utilise comme un moyen, un instrument de son propre comportement. La signification différencie l'homme de l'animal tandis qu'on trouve le processus de la signalisation chez l'animal ainsi que chez l'homme. La signalisation est une prémisse nécessaire de la signification qui, cependant, ne peut pas être une base adéquat du comportement de l'homme.

Vygotski a distingué donc les signes-signaux et les signes proprement dits. La distinction dépend du caractère de la réponse de l'homme au produit de l'activité signalétique qui lui vient d’être offert par un autre homme. Si la réponse est conditionnée et univoquement déterminée, c'est-à-dire le signal représente le stimulus d'une seule réaction, alors l'activité psychique ne tient pas de l'activité mentale car elle ne comprend pas de tâche mentale. Ce sont, selon Vygotski, les signes-signaux.
Si le produit de l'activité signalétique n’est pas déterminé - dans le cas où la réponse au signal peut être variée, peut représenter une action, un signal ou une absence significative de réponse à la stimulation signalétique - alors là ce sont, selon Vygotski, les signes proprement dits. Le signe est une variété du signal qui exige une solution d'une tâche mentale. La création des signes dans les activités humaines présuppose la résolution d'une tâche mentale.

Ainsi, selon la théorie de Vygotski, l'homme ayant reçu un signe-signal, y répond toujours et obligatoirement par une action qui lui est imposée par ce signal. Ayant reçu et compris le signe proprement dit, l'homme n'est pas obligé à y répondre par une action quelconque. Dans ce cas la réaction peut être triple: 1) répondre par une action ou par une série d'actions variées; 2) répondre par un ou par des signes; 3) labsence de réponse.

Ladaptation active de l'homme au milieu environnant demande la formation active des liaisons ce qui est impossible dans le comportement naturel. L'homme introduit des stimuli artificiels et à l'aide des signes il crée de nouvelles liaisons dans le cerveau. Il apparaît une nouvelle caractéristique du comportement de l'homme - l'autostimulation (Vygotski, 1931/1983).

\section{Symbole dans l'approche de Vygotski}

La tragédie de Shakespeare "Hamlet" accompagnait Vygotski au cours de toute sa vie. Hamlet, selon lui, est une "tragédie de toutes les tragédies". Il a commencé sa vie professionnelle par l'analyse psychologique de l'image de Hamlet entre 1914 et 1916, tout jeune, et il a fini sa vie à lâge de 37 ans à un hôpital où il avait apporté avec lui cette œuvre. Il a écrit deux travaux sur "Hamlet" avec un intervalle entre eux de 10 ans. Au cours des années, son interprétation de la tragédie a changé essentiellement. Si sa première essai sur "Hamlet" se concentre sur l'interprétation symbolique de la tragédie, ce côté de l'interprétation a été complètement éliminé dans son chapitre sur Hamlet dans la "Psychologie de l'art". On peut dire qu'avec le temps il a refusé d'analyser le côté symbolique d'un œuvre d'art ainsi qu'utiliser la notion du symbole dans son approche historico-culturelle à la psychologie.

Finalement, l'idée de la médiation du développement d'homme a été rétrécie chez Vygotski. De tous les médiateurs possibles on peut y 
trouver seulement deux : le signe et le mot. En dehors de son analyse il a laissé le symbole et le mythe.

Tandis qu'ils sont une partie intégrante du psychanalyse, le symbole et le mythe sont faiblement présentés dans la psychologie historicoculturelle, ce qui est assez paradoxal: sans symbole et mythe il est impossible de comprendre la formation de la conscience et de la personnalité. Aujourd'hui, des continuateurs de Vygotski essayent de combler ses lacunes dans la psychologie historico-culturelle (par exemple V.P. Zinchenko).

Pourquoi Vygotski a désavoué du concept du signe ? On peut supposer que c'est arrivé sur l'influence du marxisme. Ce nétait pas par hasard que Vygotski nommait les médiateurs des instruments psychologiques. Si c'est le symbole qui fait l'homme d'un être humain, il s'agit de l'idéalisme.

Si c'est un objet, il s'agit du matérialisme. En essayant dêtre un véritable matérialiste et marxiste Vygotski laisse tomber le symbole. L'hypothèse de Florenski que le passage d'in objet à une idée et à l'inverse est toujours médiatisé par un symbole a été étrangère pour le matérialisme dialectique. En même temps, les idées de Lossev sur les médiateurs (signe, mot, symbole, mythe) ont influencé Vygotski.

\section{Approche historico-culturelle et psychanalyse}

Même si Vygotski a discuté avec psychanalyse, son intérêt y a été vif. Premièrement, il n'était pas d'accord avec le symbolisme total de Freud, et deuxièmement, il a critiqué le point de vue que freudisme et marxisme étaient des systèmes voisins. "Il en résulte une situation très étrange : Freud et son école ne se déclarent jamais monistes, matérialistes, dialecticiens ou héritiers du matérialisme historique. Tandis qu’on leur annonce: vous êtes tout cela à la fois ; vous ne savez pas vous-mêmes qui vous êtes" écrit-il en 1927. En même temps, les livres de Freud ont exercé une influence visible sur ses travaux. En 1929 il a figuré sur la liste des membres de la Société psychanalytique et il avait beaucoup d’amis et de collaborateurs dans cette société. Voici ce quont écrit en 1925, Vygotski et Luria: "Chez nous, en Russie, le freudisme fait l'objet d'une attention exceptionnelle non seulement dans les milieux scientifiques, mais aussi auprès d'un large public. Actuellement, toutes les œuvres de Freud ou presque ont été traduites en russe et publiées. Nous voyons naître sous nos yeux en Russie un nouveau courant original en psychanalyse, un courant qui tente de réaliser une synthèse entre freudisme et marxisme grâce à la théorie des réflexes conditionnés" (Vygotski, Luria, 1989, p. 29.).

\section{Limites de l'approche historico-culturelle}

En analysant le rôle de l'environnement dans le développement et le comportement de l'homme, la psychologie historico-culturelle laisse, cependant, à côté une question très importante liée à l'adaptation de l'homme au milieu environnant qui change continuellement. Est-ce que l'intériorisation de normes sociales et culturelles mène toujours aux conséquences positives pour l'homme? Malheureusement, la culture a ses côtés négatifs, sombres et nous tend souvent des pièges. Les nouvelles technologies, la croissance énorme des innovations et des moyens techniques donnent naissance non seulement à des avantages mais aussi à de nouvelles formes de la pathologie. Il faut qu'aujourd'hui nous interprétions cette réalité aux termes de la théorie historico-culturelle.

Les conséquences négatives les plus évidentes de l'influence de la culture et de la société sur l'homme peuvent être suivantes:

Premièrement, ce n'est pas toujours vrai qu'une fonction médiatisée par la culture et par la société a les avantages par rapport à une fonction naturelle.

Deuxièmement, il existe un type spécifique de la "pathologie culturelle" - de différentes formes de la dépersonnalisation qui sont liées à un écart par rapport à la norme en ce qui concerne le processus de l'aliénation normale.

Finalement, en facilitant notre vie, la culture nous prépare encore un piège: l'homme cesse de faire un effort et au lieu du progrès, la culture l'amène à une régression. Par exemple, l'utilisation de la voiture mène à l'obésité, l'utilisation du calculateur par un enfant - à l'incapacité de l'utiliser du calcul mental (Tkhostov, Sournov, 2005).

Il faut qu'aujourd'hui nous interprétions cette réalité aux termes de la théorie historico-culturelle. Comme a écrit A.N. Léontiev, "Nous comprenons la conception de Vygotski non pas comme un système de dogmes qu'il reste soit à accepter soit à rejeter, mais comme la première esquisse, qui est peut-être encore imparfaite, du chemin qu'elle découvre. Et si au cours du développement de la science psychologique [...] 
beaucoup de choses seront changées et même rejetées, on verra plus clairement ce qui y est positif et indiscutable et qui compose son véritable noyau".

\section{Bibliographie}

Léontiev, A.N. (1976). Le développement du psychisme. Paris: Ed Sociales.

Tkhostov, A.S, Sournov, K.G. (2005). Vliyanie sovremennyh tehnologij na razvitie lichnosti i formirovanie patologicheskih form adaptacii: obratnaya storona socializacii [L'influence de technologies contemporaines sur le développement de personnalité et la formation de formes pathologiques de l'adaptation: le côté inverse de la socialisation]. Psihologicheskii zhurnal, 6. P. 15-42.

Vygotski, L.S. (1925/2005). Psychologie de l'art. Paris: La Dispute.

Vygotski, L.S. (1928/1991). Problema kul'turnogo razvitiya rebenka [Le développement culturel de l'enfant]. Vestnik Moskovskogo universiteta. Seriya 14: Psihologiya [La revue de l'Université de l'État de Moscou. Série 14: Psychologie], 4. P. 5-18.

Vygotski, L.S. (1931/1983). Istoriya razvitiya vysshikh psihicheskikh funkcij [Histoire du développement des fonctions psychiques supérieures]. Dans Sobranie sochinenii $v 6$ t. [CEuvres en 6 volumes]. Vol. 3. Moskva: Pedagogika. P. 5-328.

Vygotski, L.S. (1933/1985). Le problème de l'enseignement et du développement mental à lâge scolaire. Dans B. Schneuwly, \& J.-P. Bronckart (eds.) Vygotsky aujourd'hui. Neuchâtel: Delachaux et Niestlé. P. 95-117.

Vygotsky, L.S. (1998). Théorie des émotions: étude historico-psychologique. Traduction de Nicolas Zavialoff et Christian Saunier. Introduction de Nicolas Zavialoff. Paris, L'Harmattan.

Vygotski, L.S., Luria, A.R. (1989). Predislovie k russkomu perevodu raboty Frejda "Po tu storonu principa udovol'stviya" [Préface à la traduction russe de "Au-delà du principe du plaisir"]. Dans S. Freud. Psihologiya bessoznatel'nogo [Psychologie de l'inconscient]. Moskva: Prosveshenie.

Zinchenko, Y.P., Tkhostov, A.S., Shilko, R.S. (2008). Filosofskie i metodologicheskie problemy psihologii [Philosophical and methodological problems of psychology]. Dans Filosofiya social'nykh i gumanitarnykh nauk [Philosophy of social and humanitarian sciences]. Moskva: Akademicheskij proekt. P. 608-652. 